Original Research Paper

\title{
Smart-Factory: Optimization and Process Control of Composite Centrifuged Pipes
}

\author{
${ }^{1}$ Raffaella Aversa, ${ }^{2}$ Relly Victoria V. Petrescu, ${ }^{2}$ Florian Ion T. Petrescu and ${ }^{1}$ Antonio Apicella \\ ${ }^{1}$ Advanced Material Lab, Department of Architecture and Industrial Design, \\ Second University of Naples, 81031 Aversa (CE) Italy \\ ${ }^{2}$ ARoTMM-IFToMM, Bucharest Polytechnic University, Bucharest, (CE) Romania
}

Article history

Received: 29-10-2016

Revised: 24-11-2016

Accepted: 29-11-2016

Corresponding Author:

Florian Ion T. Petrescu

ARoTMM-IFToMM, Bucharest

Polytechnic University,

Bucharest, (CE) Romania

Email: scipub02@gmail.com

\begin{abstract}
Process optimization strategies and intelligent control algorithms for an industrial centrifugal pipe production are presented. The chemorheological and thermo-kinetic features of the polymerization of unsaturated polyester based composites are analyzed by means of a mathematical model that uses the heat transfer and generation properties of polymerizable systems. The evolution of the system temperature, viscosity and degree of cure profiles in a composite centrifuged pipe wall have been identified and mathematically described. The temperature profiles were calculated according to an appropriate kinetic and heat transfer modeling and then the corresponding viscosity profiles were evaluated. The viscosity and kinetic parameters to use in the model were measured in calorimetric and rheological tests. A commercial polyester system, which is used for centrifugal forming, has been catalyzed with two different types of peroxide (Methyl-Ethyl-Ketone and Acetyl-Acetone peroxides) and characterized by Differential Scanning Calorimetry (DSC) and adiabatic peak temperature measurements (gel time tests). The problem of incomplete cure in sections of the pipe wall has been discussed with respect to the processing conditions and creep behavior. Experimentally verification of thermal theoretical modeling and creep tests have been carried out on a system catalyzed with AA peroxide and processed in two different ways. The thermo-kinetic process modeling and control adequately predicts the thermal behavior at the internal surface of pipes that has been used to optimize by simulation of two different centrifugal pipe processing thermal cycles.
\end{abstract}

Keywords: Smart Factory, Centrifugal Pipe, Process Control, Glass Reinforced Plastics, Chemo-Rheology

\section{Introduction}

Currently we are standing on the edge of a fourth industrial revolution; one which, while enabling us to mate the worlds of production and the "Internet of Things", is making "Industry 4.0 " a reality that could connect embedded system production technologies and smart production processes.

The development of the new technological age of the "Smart factory" will radically transform the industrial production value chains and business models. "Smart production" is then becoming the standard in a world where ICT intelligent machines, networks and systems are capable of independently exchange and respond to information to manage an industrial production processes (Hwang et al., 2016).
The present paper presents an experimental verification of the application of an intelligent process strategy choice and control for the fabrication of Glass Reinforced Polymeric (GRP) centrifuged pipes.

Flexible GRP pipes are designed to act as soil-pipe interaction system and, as such, the external loadings resulting from the soil pressure, assembly, accidental loads and impacts must be evaluated for this type of design system to perform properly.

The manufactured product must maintain, from a quality control and design point of view, a constant and adequate smooth wall thickness and profile. Any variance from the theoretical thickness or not homogeneous profile alters the pipe stiffness values leading to:

- Increased deflection 
- Increased dependence on soil to carry the load and minimize deflection

- Decreased resistance to buckling

- Increased distortion of the pipe profile

- Increased strain in pipe wall

- Joint leaks

- Reduced hydraulic capacity

- Ground surface settlement

- Increased aging by stress corrosion

The factors that affect GRP pipe stiffness are:

- $\quad$ Stress and Strain distribution

- Ratio External Temperature/Glass transition of the polymerized resin

- Creep characteristics (viscoelasticity)

- Manufacturing flaws and tolerances

- Use of fillers and their positioning in the pipe wall

- Type of thermosetting resins

During the processing of thermosetting-based composites, shaping operations are accompanied by polymerization reactions and rheological changes that, altering some of the feature of the previous list, may strongly influence the quality and the final mechanical properties of the composite structure.

Pioneering studies of the chemo-rheological behavior prediction has been done in the 80's (Apicella et al., 1983; 1984; Nicolais and Apicella, 1985; Kenny et al., 1989).

In these studies by Apicella et al. $(1983 ; 1984)$ it has been mathematically described and links together the chemical and physical phenomena occurring during polymerization of thermosetting resins. The initial stages of the polymerization process of a composite pipe are associated with a significant increase of the material viscosity (molecular weight increase) that is combined with a heat generation due to the exothermic nature of the polymerization reactions. The relative rates of heat generation and heat transmission govern the temperature through the pipe wall and therefore, the reaction conversion and the resin viscosity through the thickness of a composite part.

An uncontrolled polymerization may cause excessive undesired variations of the temperature that could induce in homogeneity in the polymer network and macroscopic defects in the composite (voids, bubbles and debonded and broken fibers (Halpin et al., 1983; Kenny et al., 1989). Processing of polymeric composites based on thermosetting matrices needs, therefore, optimization of the cure cycle parameters as well as adequate formulation of the reacting system as a function of the geometry of the part (Bamford et al., 1958; Beisemberg and Sebastian, 1983; Odian, 1970; Huang and Lee, 1976; Han and Lem, 1983; Han and Lee, 1987).
Polymeric composite pipes that are used in water and sewage transport are made of glass reinforced unsaturated polyester (GRP). The final properties of such pipes depend on the manufacturing method. Low pressure applications pipes could be made by centrifugal casting, a technique in which chopped fibres and liquid resin are pored together inside of a rotating steel cylindrical mold by centrifugal force until the resin has set. For high-pressure applications, conversely, the use of continuous glass fibers by filament winding these fibers onto a rotating mandrel could attain high mechanical strength and rigidities.

An advantage of the centrifugal casting production method over the filament winding is that one (higher) filler content can be achieved. Moreover, the addition of siliceous sand, Calcium carbonate or other filler to thicken the walls will increase the pipes rigidity in radial compression since wall stiffness is a function of the cube of its thickness. Finally, by varying the amount, proportions and orientation of the cut fibers and particulate fillers through the pipe layers, different final mechanical properties can be obtained both for low pressurized or unpressurised applications. The steel rotating molds can be thermo-stated by water circulating jacket or internally heated by hot air flux in order to reach a suitable temperature ensuring the correct polymerization kinetics and levels. Excessively high or low kinetics of polymerization could lead to an uncontrolled temperature rise or incomplete resin polymerization. The unsaturated polyester resin polymerization process should be then finely monitored and controlled if constant and reliable quality (of the GRP pipes) is needed.

\section{Materials and Methods}

\section{Materials}

The systems analysed in our case study is a commercial grade unsaturated orthophtalic polyester used as matrix for the structural part of glass reinforced pipes made by centrifugal forming. The resin was activated with $0.5 \%$ of Cobalt Octoate and $1.5 \%$ of two different peroxides, namely, Methyl-Ethyl-Ketone Peroxide (MEKP) and Acethyl Acetone Peroxide (AAP).

\section{Apparatus and Procedures}

\section{Differential Scanning Calorimetry}

The thermo calorimetric characterization has been carried out in a Mettler ADSC Differential Scanning Calorimeter equipped with a liquid nitrogen cooling unit in the range of temperatures between 0 and $300^{\circ} \mathrm{C}$, in a nitrogen atmosphere. Temperature scans were carried out at $5^{\circ} \mathrm{C} / \mathrm{min}$. Isothermal scan were run at 20,30 , $40,50,60$ and $70^{\circ} \mathrm{C}$, heat flux were recorded up the final apparent equilibrium (heat flux $=0$ ). 


\section{Dynamic Thermo-Mechanical Characterization}

Dynamic-mechanical shear tests on the resins during the polymerization have been done by using a Mettler Dynamic Viscoelastic Analyzer (DMA). Samples were tested in shear load control mode at a frequency of $8 \mathrm{~Hz}$ by imposing loads of 0.5 and 0.05 $\mathrm{MPa}$ static and dynamic mode, respectively. Viscoelastic response data were measured in shear load control mode during the isothermal pause of $20 \mathrm{~s}$ with temperature step of $10^{\circ} \mathrm{C}$ with an overall heating rate that was set both at $5^{\circ} \mathrm{C} / \mathrm{min}$.

Reaction kinetics for the resin catalysed systems, specific heat, final glass transition temperatures and the level of polymerization reached by the resins after the process were determined.

\section{Static Mechanical Characterization}

Creep test were performed on pipes DN600 (600 mm diameter). Pipe stiffness for GRP pipe was evaluated as the inherent strength measured in accordance with ASTM D 2412 Standard Test Method for the determination of the characteristics of an external loading of Plastic Pipe by using parallel plate set at the industry standard of $5 \%$.

\section{Pipe Centrifugal Processing}

An automatic and electronically controlled centrifugal casting system has been adopted. Controlled material qualities (poured in the mould layers by layers), mould rotation speed and internal temperatures were closely regulated to achieve the designed result moulded pipe. Glass fibre, polyester resin and silica sand are fed into the pipe rotating mould working inwards until the required wall thickness is obtained.

The analysis of the resin reaction kinetics has been carried out to define the correct formulations (initiator and inhibitor concentrations) so that polymerization start only after complete filling had taken place (induction time).

The reinforcing glass filaments, pre-cut to the 1.5-2.0 $\mathrm{cm}$ length, are introduced from the head of a feeder arm which moves back and forth inside the mould distributing the fibres within two high strength layers to provide the designed circumferential and axial strength and elastic modulus. Siliceous sand layers were used in the central core, which was intended to increase the pipe wall thickness and in the outer pipe wall surfaces, which was aimed to increase the polyester resin matrix resistance and resilience, while a resin rich layer was poured in the inner layer to create a smooth pipe internal surface.

The mould was initially slowly rotated (10-20 rpm) at start and then rotation speed was progressively increased up to $100 \mathrm{rpm}$ once the raw materials were introduced and the polymerization started. This operation ensured an adequate set and compaction of the fillers while polymerization was taking place.

\section{Polymerization Chemical Behavior}

Commercial resin systems were supplied as relatively low molecular weight unsaturated aromatic polyesters (orthophthalic) dissolved in a polymerizable monomer (styrene) providing crosslinking units by reacting with the unsaturation of the polyester resin in a radical addition reaction. Finally, initiators, inhibitors and other additives are added to the resin monomers and oligomers to control the reaction kinetics (induction time and reaction times), (Fan and Lee, 1986).

The complex chemistry of the unsaturated polyester reactions has been largely reported in the literature (3-6). First, the initiator is decomposed leading to the formation of free radicals that progressively reduce the inhibitor concentration (Flory, 1953). The cure reactions start only after an induction time needed to exhausts the inhibitor then the unsaturated polyester and styrene radicals are first formed and grown to a larger size. The termination reactions progressively reduce the active radical concentration until polymerization slows and then stops (Lee, 1981).

Differential Scanning Calorimetry has devoted the present research to the evaluation of the kinetics of the curing parameters of thermo settings polyesters (Lee and Han, 1987).

Even if in most of the commercial radical thermosetting systems one approach considering an nthorder equation with a temperature dependent rate constant $\mathrm{K}$ given by an Arrhenius expression, more complex alternative expressions can be also adopted in the definition of the process control algorithm.

Pusatcioglu et al. (1979) used the following kinetic model to describe the polymerization rates of isothermal and dynamic calorimetric experiments:

$$
\frac{d W}{d t}=A \exp \left(\frac{E a}{R T}\right) W^{a}(1-W)^{b}
$$

where, $d W / d t$ is the reaction rate, $W$ is the extent of reaction, $T$ is the absolute temperature (in ${ }^{\circ} \mathrm{K}$ ), a, b are two temperature independent constants, $A$ is the rate constant and $E_{a}$ is the activation energy of the curing reaction (in their case about $17 \mathrm{Kcal} / \mathrm{mol}$ ).

Kamal et al. (1973; Han and Lee, 1987) proposed a slightly more complex kinetic model:

$\frac{d W}{d t}=\left(A_{1}+A_{2} \cdot W^{a}\right) \cdot(1-W)^{b}$

While Cuadrado et al. (1983) interpreted the DSC data through two different regimes: At low temperatures, the reaction rate shows a first order kinetic with activation energy of $15 \mathrm{Kcal} / \mathrm{mol}$, while at high temperatures $\left(\mathrm{T}=100^{\circ} \mathrm{C}\right)$ a change in the kinetic regime was detected. 
The following expression was obtained from experimental data at high temperature:

$$
\frac{d W}{d t}=5.2 \cdot 10^{26} I_{0} e^{\left(-\frac{48.5}{R T}\right)} \cdot(1-W)^{2}
$$

where, $I_{0}$ was the concentration of the initiator (in their case benzoyl peroxide).

\section{Rheological Behavior}

The branching and crosslinking reactions between unsaturated polyester double bonds and styrene strongly increase the resin viscosity. As already discussed in previous publications (Kenny et al., 1989; Kaštánek and Bohdanecký, 1985), the rheological behaviour of a polymerizing resin is headed by two phenomena: The first associated to the molecular structural changes induced by the polymerization reactions and the second one associated to the variation of the segmental mobility determined by temperature variations induced by heat accumulation following the exothermic reaction.

The theoretical molecular weight dependence of the viscosity for different polymeric systems has been described in literature (Kenny et al., 1989; Kaštánek and Bohdanecký, 1985; Apicella, 1986).

A William-Landel-Ferry type equation was proposed by Apicella et al. (1984) to represent the rheological changes during the cure of an epoxy/amine system used as a matrix of high performance composites. This model contains molecular parameters (such as molecular weight or branching coefficient) as well as physical parameters such as temperature and glass transition temperature). In the case of polyester radical polymerization, however, this model cannot be directly applied. However, the rheological modifications accompanying the radical polymerization of complex unsaturated polyester mixtures can be described by empirical expressions such as those proposed by Castro and Macosko (1982). The following relationship between the viscosity, the temperature and the degree of reaction for polyurethane systems was adopted in our radical polymerizations processes:

$\eta=K_{\eta} e^{\left(E_{\eta} / R T\right)}\left[W_{g} /\left(W_{g}-W\right)\right]^{(A+B W)}$

where, $K_{\eta}$ is a pre-exponential factor, $E_{\eta}$ is the activation energy for the viscosity changes, $\mathrm{A}$ and $\mathrm{B}$ are constants and $W_{g}$ is the extent of reaction at the gel point.

\section{Development of the Thermo and Chemo- Rheological Model}

In previous experiences we developed an algorithm for the description of the chemo rheological behaviour during a lamination process of epoxy-based systems
(Kenny et al., 1989). The algorithm, based on the local energy balance, was applied to the estimate of the thermo-rheological behaviour in a plane glass fibber composite undergoing different process conditions.

The curing behaviour of unsaturated polyester resins is characterized by a more complex mechanism involving copolymerization of the polyester and styrene produced by the radical decomposition of the initiator. In order to optimize and properly control the cure process, different resin thermo-kinetics and rheological models are to be used in the computer process simulation algorithm.

In particular, the algorithm involves the solution of an energy balance, which takes into account the accumulation of heat in the bulk composite, the heat generated by the exothermal chemical reaction, the heat conduction in the material and the heat dissipation at the composite skin boundary conditions. The energy balance equation is coupled with a suitable expression for the kinetic behaviour of the chemical reactions. The solution of the complete mathematical system gives the temperature and the degree of reaction evolution as a function of time and position using, as input data, the experimental results of the resin thermo-calorimetric and rheological characterization. In parallel, the rheological behaviour of the polyester matrix is then described by using the mathematical model to compute the viscosity as a function of the temperature and the degree of reaction.

\section{Process Monitoring and Control Algorithms}

The use of the thermokinetic and chemo rheological models leads to the computation of viscosity profiles during the polymerization once temperature and resin conversions are calculated. When the process may be considered isothermal, i.e., for thin composite, the degree of cure at different times can be computed solving the appropriate kinetic model. Nevertheless, as previously discussed, the cure of a polyester resin is always coupled with a very rapid and sensible development of heat that is not easily dissipated leading to sensible temperature rises and consequent reaction acceleration (which further increases the heat generation rate). The profiles of temperature and degree of cure in the composite must be calculated taking into account the system geometry, the thermal diffusivity of the composite and the resin reaction rate. By solving in parallel the energy balance with an appropriate expression for the cure kinetics, this task can be achieved by using existing mathematical solvers software (Kenny et al., 1989; Pusatcioglu et al., 1979).

Special attention has been devoted in this study to the effect of the induction time that is an integral and critical part of the centrifugal pipe processing.

In the first stage of the process, when reaction are still controlled by the inhibitor, the temperature inside of the composites is gradually increased only by the effect of the heat transferred by conduction from the 
internal and external pipe surfaces (boundary conditions). The induction phenomenon, which is an activated process, is influenced by these temperature rises and should be evaluated for all time and positions through the pipe thickness (namely, this means that the polymerization will start at different times through the pipe wall). The induction time variations at each position and time may be computed at a certain temperature by using Equation 5. When the induction time in a particular pipe section reaches zero, the reaction will start. In non isothermal process this condition can be represented for each point or the composite by the following equation:

$$
\int_{0}^{t} d t / t_{i 0} e^{\left(\frac{E_{t}}{R T}\right)}=1
$$

By including the effect of the induction in the model two balances must be written. For a plane composite or large diameter pipe wall, the system of equations in dimensionless form, before the start of reaction, is:

$$
\frac{d \theta}{d t^{*}}=D e \frac{d^{2} \theta}{d X^{2}}
$$

where, $D e$ is the Deborah number:

$$
D_{e}=t_{g} k_{x} /\left[C_{p}(h / 2)^{2}\right]
$$

De corresponds to the ratio of the characteristic time associated to the transition originated in the polymeric structure by the crosslinking chemical reactions (isothermal gel time $t_{g}$ ) and the characteristic time of the heat diffusion $\left.C_{p}(\mathrm{~h} / 2)^{2} / k_{x}\right)$.

Once the reaction starts it can be written:

$$
\begin{aligned}
& \frac{d \theta}{d t^{*}}=D_{e} \frac{d^{2} \theta}{d X^{2}}+S t^{d D_{e}} / d t^{*} \\
& \frac{d W}{d t^{*}}=K^{*} e^{-\frac{1}{\left[\theta\left(T_{e}-T_{0}\right)+T_{0}\right](1-W)^{n}} *^{\frac{E_{a}}{R}}}
\end{aligned}
$$

Temperature $\theta=\left(T-T_{0}\right) /\left(T_{e}-T_{0}\right)$

Time $t^{*}=t / t_{g}$

Position $X=x /(h / 2)$

The use of the Deborah number in the description of the behaviour of reactive polymer systems has been reported previously (Kenny et al., 1989). De represents the relationship between the heat transferred by conduction and the heat stored in the material. For glass fiber/polyester resin composites, obtained by RTM or pultrusion, typical De numbers are of the order of 10 . Also for carbon fiber/epoxy resin laminates, typical $D e$ numbers are of the order of 10 (Kenny et al., 1989).

The isothermal gel time $\left(t_{g}\right)$ is used as a characteristic time to describe the structural changes produced by the crosslinking reactions and it can be calculated by integration of the kinetic Equation 6:

$t_{g}=\frac{1}{K_{0}} e^{\left(\frac{E}{R T}\left[\left(\left(1-W_{g}\right)^{1-n}-1\right) /(n-1)\right]\right.}$

In Equation 9 the Stefan Number, St, is also introduced:

$S t=\frac{H_{r}}{\left(T_{e}-T_{0}\right) C_{p}}$

It may be considered as the ratio between the latent heat associated to the chemical reaction and the accumulation of heat in the material. Typical Stefan numbers for these processes are of the order of 1 .

In Equation 9 a dimensionless kinetic constant $K^{*}$ is used and the following expression for $K^{*}$ is derived from Equation 1 and 13:

$K^{*}=\left[\left(1-W_{g}\right)^{1-n}-1\right] /(n-1)$

Typical values for $K^{*}$ were found of the order of 1 .

When the top $T_{h}$ and the bottom $T_{i}$ surface temperatures are equal to the external process temperature $\left(T_{e}\right)$ and that the initial temperature $\left(T_{0}\right)$ is constant across the laminate, the dimensionless initial and boundary conditions become:

Dimensionless initial conditions:

$t^{*}<0 \mathrm{~W}=0$ and $\theta=0$

Dimensionless boundary conditions:

$t^{*} \geq 0 ; X=0$ and $d \theta / d X=0$

$X=1 \quad \theta=\theta_{1}\left(t^{*}\right)$

Furthermore Equation 5 must be considered as an additional boundary condition for the determination of the starting time of the polymerization reactions.

Once the temperature and degree of reaction are known, the resin viscosity can be calculated as a function of position and time.

For the numerical solution of the mathematical algorithms illustrated, Mathlab (Mathworks) was utilised while for monitoring and active control, Arduino platform software was interfaced in this first monitoring and process control trial to modify a relevant processing 
parameters such is the heating time given by the external hot water jacket of the rotating mould.

\section{Results and Discussion}

We have analyzed the processing conditions of two different production lines of glass reinforced pipes obtained by centrifugal forming. In particular, the preliminary investigated processes were characterized by different heating procedures; from inside with hot air blowing $\left(100-120^{\circ} \mathrm{C}\right)$ and from outside with one waterjacket $\left(70-80^{\circ} \mathrm{C}\right)$. Moreover, two different initiators were used (MEKP and AAP) for the polymerization of the unsaturated polyester.

Due to the higher reliability of the heating system, the water jacked controlled systems was chosen against the hot air flow and it has been used in the trial tests of process monitoring and control.

Nevertheless, even for the pipe processed with the water jacket in the ordinary factory condition without the proposed control and monitoring system, the glass transition temperatures were not easily predictable, namely they were about $70^{\circ} \mathrm{C}$ for the material processed for $5 \mathrm{~min}$ and only $80^{\circ} \mathrm{C}$ for the material heated three times longer (15 min).

Moreover, in such condition of high variability of the polymerization level, the pipe stiffness could be very variable do to the increased probability of undergoing higher creep for the less polymerized resins. It was then mandatory to explore and predict what would be the temperature and conversion profiles through the pipe thickness.

We then started with the resin thermo calorimetric characterization to achieve the kinetic parameters to use in the model.

Dynamic and Isothermal thermal scan using the Differential scanning calorimeter was carried out on differently activated thermosetting resins. Moreover, an adiabatic apparatus for the measure of the increase in temperature during the polymerization (gel time test) at different initial temperatures were also carried out.

The following describes the tests performed and the parameters measured:

- Adiabatic gel time test

- Measurements of the induction and reactivity from gel time and DSC tests Gel Time Tests

- Dynamic DSC themal scans

- The overall heat of polymerization $\mathrm{Hr}$, of the system catalyzed with MEKP and AAP

- The specifically heat of the resin and of the glass reinforced material

- Isothermal DSC themal scans

- The polymerization kinetic parameters (reaction order, activation energy and kinetic constant) at different temperature in order to evaluate their temperature dependency
- The induction times at different isothermal temperatures in order to define the Gel time/Temperature relationship (in the Arrhenius form)

- The pre-exponential factor of the Arrhenius equation when an inhibitor is added to the reacting system

\section{Adiabatic Gel Time Test}

The gelation and reactivity characteristics of the resin formulations (type of resin, amount of catalist, initiator and inhibitor) were analyzed by measuring the increase of the temperature of a know amount of resin undergoing polymerization (gel time test) in adiabatic like condition as indicated in Fig. 1.

As previously discussed, the measured temperatures and their kinetics of variation are strongly dependent on the thermal exchange conditions. In the same conditions of thermal exchange, a system of lower reactivity will produce an apparently lower increase of the temperature of a faster system due to the higher heat losses even if the overall heat of reaction is the same for both systems.

The increase of the gel times is achieved by using inhibitors. The reactivity (the time needed to complete the reaction once started) of a system containing an inhibitor should remain the same as the system without inhibitor since these chemicals are acting only the reaction starting time. However, in conditions where the heat exchange is overcoming the effect of internal heating due to the polymerization, the effect of the inhibitor can be masked and not evaluated correctly.

Due to the high heat generation rate compared to the heat losses, fast reacting systems may tend to show an adiabatic like behavior even when not well insulated. The system will tend to behave adiabatically when, for all reaction rates, the heat generation is fast compared to the rate of heat loss; this can be achieved by well insulating the reacting system during the gel time test.

In order to perform the gel time tests in similar conditions for different reactivity systems, it has been chosen to insulate the reacting system during the test. For all these reasons, we carried out this test in adiabatic like conditions. In these conditions, in fact, the test will be independent of the reactivity of the resins. Moreover, information's on the gel times and reactivity, as defined by this test, can be compared to systems catalyzed with different amounts and types of peroxides.

An evaluation of the overall heat of reaction can be obtained from the knowledge of the real temperature rise due to the exothermic reaction once the specific heat of the material is known.

In this test, in fact, the adiabatic increment of temperature is given by the expression $\Delta \mathrm{T}=\mathrm{Hr} / \mathrm{Cp}$

\section{Definition, Measurements and Prediction of the Gel Times}

The delay in the initial thermal behavior, which is due to the competition of initiation and inhibition 
reactions, is temperature dependent and will be treated as an activated process. The rheological analysis of the systems investigated was an oscillatory shear experiment with a DMA tester operating at $1 \mathrm{~Hz}$ and it was finalized to the evaluation of the isothermal complex viscosity and loss factor as a function of time. After the reaction time lag described earlier, the resin viscosity begins to grow as a consequence of the increase of its average molecular weight and of its molecular complexity. The loss factor (the ratio between the viscous and elastic components of viscosity) depends on the macromolecular modifications and it varies from values higher than 1 (viscous behavior) to values lower than 1 (elastic behavior). During the polymerization, in fact, once the gel point is overcome, the material changes from a viscous liquid to a rubber.

The gel point of the resin may be identified by the minimum of the loss factor curve (when the elasticity is prevalent) accordingly to independent constant shear measurements performed under the same conditions (when the viscosity abruptly reached practically infinite values).

By comparing the kinetics of viscosity variation with the conversion kinetics determined in the calorimetric tests, the characteristic critical gel conversions used in the model were calculated. In unsaturated polyesters, gel point generally occurs at very low conversions (less than $8 \%$ ) just after the polymerization reaction starts.

Gelation has been considered for these systems to be almost coincident with the induction time.

Gel times and reactivities obtained from the gel time curves run in adiabatic like conditions (Fig. 1) are reported in Table 1 for MEKP and AAP catalyzed resins.

As indicated in Table 1, the system catalyzed with AAP present a reactivity (which is inversely proportional to the time necessary to reach the peak temperature) that is significantly higher than that of the system using MEK, 7 and 13 min respectively. Moreover, as expected, the values of reactivity are not influenced by the presence of the inhibitor while gel times are strongly modified; from 11 to $21 \mathrm{~min}$ for MEKP systems and from 8 to $14 \mathrm{~min}$. for AAP systems.

\section{DSC Analysis}

In our case, in order to develop a general program for the control and optimization of the cure process of polyester based composites, a simple kinetic model has been adopted to describe the polyester crosslinking. Isothermal DSC experiments have been carried out on both polyester systems. The thermo grams analyzed showed a typical single peak thermal evolution and they have been used to compute the induction time and the kinetic parameters at different temperatures. As indicated before, the kinetic analysis of the isothermal DSC scans has been performed using an nth-order expression containing a rate constant $\mathrm{K}$ that depends on temperature according to an Arrhenius form:

$$
\frac{d W}{d t}=K(1-W)^{n}
$$

Where:

$$
K=K_{0} e^{\left(-E_{a} / R T\right)}
$$

This type of equation has been commonly adopted for other reactive systems $(2,15)$, although it can result in an over-simplified expression of the complex cure reactions occurring in most of the commercial systems (Kenny et al., 1989), this equation is able to correctly fit the experimentally observed heat of reaction releases as measured by the Differential Scanning Calorimetry.

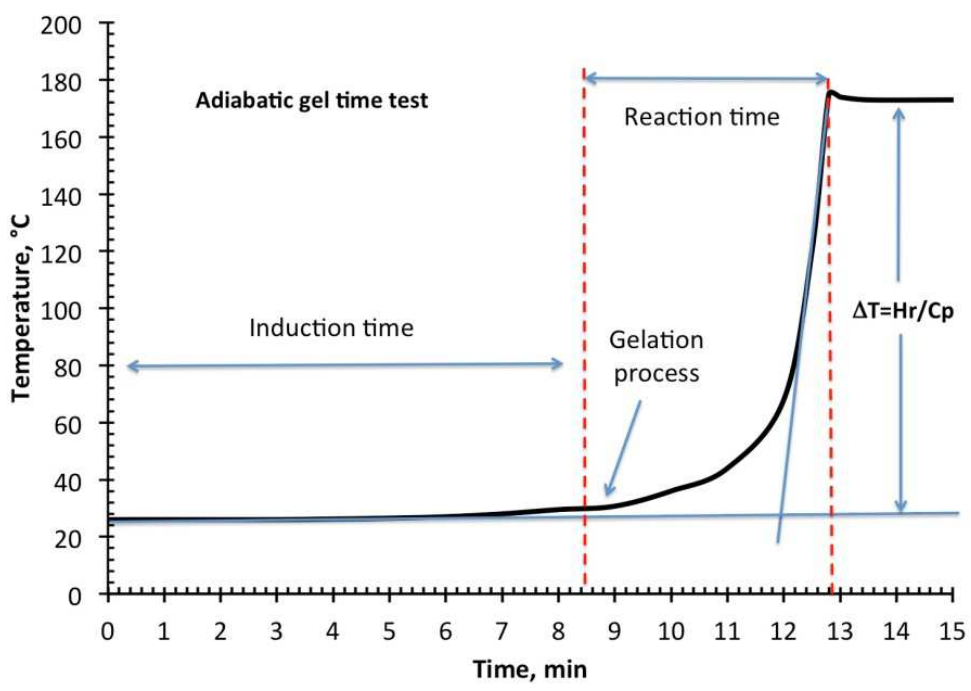

Fig. 1. Schematic Temperature-time diagram and definitions in adiabatic gel time test for thermosetting resins 
Table 1 . Gel Times at $25^{\circ} \mathrm{C}$ and reactivity of resins activated $(0.5 \%$ Cobalt octoate) e catalyzed with MEKP and AAP (1\%) in presence or not of the inhibitor $(0.5 \%)$

\begin{tabular}{llll}
\hline System & Gel time, $\min$ & Reactivity, min & Peak Temp. ${ }^{\circ} \mathrm{C}$ \\
\hline MEKP & 11 & 13 & 185 \\
MEKP + inhibitor & 21 & 14 & 181 \\
AAP & 8 & 7 & 169 \\
AAP + inhibitor & 14 & 7 & 170 \\
\hline
\end{tabular}

Table 2. Kinetic parameters for Resins catalyzed with MEK and AA

\begin{tabular}{llll}
\hline System, Catalyst & $\operatorname{lnK} 0(1 / \mathrm{s})$ & $\mathrm{Ea}, \mathrm{Kj} /$ mole & $\mathrm{n}$ \\
\hline$M E K P$ & 8.04 & 39.40 & 0.70 \\
$A A P$ & 6.44 & 33.75 & 0.75 \\
\hline
\end{tabular}

The results of the isothermal kinetic analysis of Differential Scanning Calorimetry performed on the two systems are presented in Table 2. In particular, from the values of the kinetic constants at different temperatures it has been possible to evaluate the pre-exponential kinetic factor $(\operatorname{lnk}$,$) , a mean reaction order (n) and the activation$ energies (Ea) of Equation 7 for the systems catalyzed with Methyl-Ethyl-Ketone peroxide (MEK) and Acethyl-Acetone peroxide (AA).

The Heat of reaction measured on the two systems with MEK and AAP with and without inhibitor values of the order of $300-330 \mathrm{~J} / \mathrm{g}$. The heat of reaction for a composite with $50 \%$ by weight of fillers is $150-160 \mathrm{~J} / \mathrm{g}$. From the DSC dynamic thermal scans (heating at $5^{\circ} \mathrm{C} / \mathrm{min}$ ) it was already evident that the system with AAP was more reactive, however, isothermal tests at 30 and $50^{\circ} \mathrm{C}$ were carried out in the calorimeter on the resin catalyzed with MEKP and AAP with and without inhibitor.

The induction times when AAP was present were $4 \mathrm{~min}$ at $30^{\circ} \mathrm{C}$ and $1 \mathrm{~min}$ at $50^{\circ} \mathrm{C}$. When MEKP was used, the induction times were $11 \mathrm{~min}$ at $30^{\circ} \mathrm{C}$ an $1.5 \mathrm{~min}$ at $50^{\circ} \mathrm{C}$.

The induction times in presence of AAP were, respectively at 30 and $50^{\circ} \mathrm{C}$, of 4 and 1 min while they were 11 and $1.5 \mathrm{~min}$ when MEKP was used. The presence of inhibitor increased the gel time at $30^{\circ} \mathrm{C}$ from 4 to $7 \mathrm{~min}$ for the system with AAP and from 7 to 18 min for the system with MEKP.

The induction times can be detected in calorimetric or even from gel time experiments. The gel time test, as structured, is more indicative of the induction time than gelation which can be carefully detected only with viscoelastic measurements. However, as already discussed, gelation readily follows the starts of reaction and, hence, gelation time and time to start of reaction can be considered equivalent in most practical situations.

The procedure for the evaluation of the preexponential factor to be used in the algorithm follows. Assuming that the reaction delay is governed by temperature activated processes, the induction times in the mathematical treatment is described as:

$\mathrm{t}_{\mathrm{i}}=\mathrm{t}_{\mathrm{i} 0} \exp ($ Eat $/ \mathrm{RT})$ where, $t_{i 0}$, is a pre-exponential factor which depends on the system composition and Eat is the activation energy of the process (in our case the inhibition reaction).

Once values for the induction (or gel) times at different temperatures are determined and the relationship between induction time and temperature for a system of fixed composition is established, the values of the parameters present in Equation 20 can be calculated; in our case they were $\ln t_{i 0}=-14.15$ and $E_{a t}=-41.02 \mathrm{Kj} / \mathrm{mole}$.

Any change of induction times due to the use of an inhibitor or a change in the type of peroxide can be evaluated. In fact, the activation energy of this process should not depend on these changes and the choice of the right pre-exponential factor could account for its variation with temperature.

If the gel time at room temperature $\left(25^{\circ} \mathrm{C}\right)$ is determined in adiabatic gel time experiment, then the equation can be used to determine the pre-exponential factor $t_{i o}$ to use in the algorithm proposed, which will account of the induction time variation within the pipe wall thickness were different temperature are experienced by the resin as a function of time and relative position.

\section{Evaluation of the Specific heat $C p$ of the Glass Reinforced Resin}

The value of the specific heat and thermal conductivity are necessary to model the thermal characteristic of the system in which exothermic reactions and heat transfer occur. Thermal conductivity data were taken from literature while specific heat measurements were carried out in the DSC. The value of the specific heat in the range of temperature between 20 and $70^{\circ} \mathrm{C}$ range from 2.0 to $2.2 \mathrm{~J} / \mathrm{g} 0 \mathrm{~K}$.

\section{Modeling of the Viscosity Changes}

The influence of the temperature on the viscosity was described for these polyester systems by a simple Arrhenius type equation. The initial viscosities for the unreacted systems at different temperatures were used to evaluate activation energies for the two systems En, which were evaluated to be a $16 \mathrm{kcal} / \mathrm{mol}$ (comparable with the values reported by (Cuadrado et al., 1983; Pusatcioglu et al., 1979)).

The relationship between viscosity and degree of cure has been investigated by using data obtained from isothermal experiments on the degree of cure as a function of time and on the viscosity as function of time. The variation of the viscosity as a function of the degree of cure has been described by using the 
empirical model of Equation 4. By regression analysis of the rheology experimental data the parameters of the model have been computed. The final form of the rheological model is given by:

$$
\eta=K_{\eta} e^{(-21.8+16 / R T)}[0.088 /(0.088-W)]^{(3.71-34.6 W)}
$$

In spite of the simplicity of the empirical model, the agreement between experimental data and the prediction is generally satisfactory and it is applicable also to several other unsaturated polyester systems.

\section{Use of the Algorithm for the Evaluation of the Processing Conditions}

The thermo-kinetic and chemo-rheological data, as well as the initial, internal and external temperatures and wall thickness have be input as processing data.

Temperature, polymerization advancement and viscosities have been evaluated as a function of time and position in the pipe.
Figure 2 shows the result relative to the temperature profiles generated through the pipe wall thickness during the process at different times (curves are shown by one minute steps).

It is interesting to note from Fig. 2 that the temperature gradient at the external surface, where water at $75^{\circ} \mathrm{C}$ is circulated, is directed outward after only $6 / 7$ min; this means that the system is transferring part of the heat generated by the polymerization into the thermostating water.

The heat loss lowers the temperature that the system could reach. Moreover, due to the heat transfer resistance, the temperature changes are seen by the internal surface (where measurements were experimentally taken) with a delay that in our case is of about 3/4 min.

In order to increase the internal self-heating of the resin system, it would be the case, hence, of finding the correct time to suspend the water circulation much before $20 \mathrm{~min}$ (the time used in the simulation).

Calculated internal pipe wall surface temperatures variations as a function of time are shown in Fig. 3.

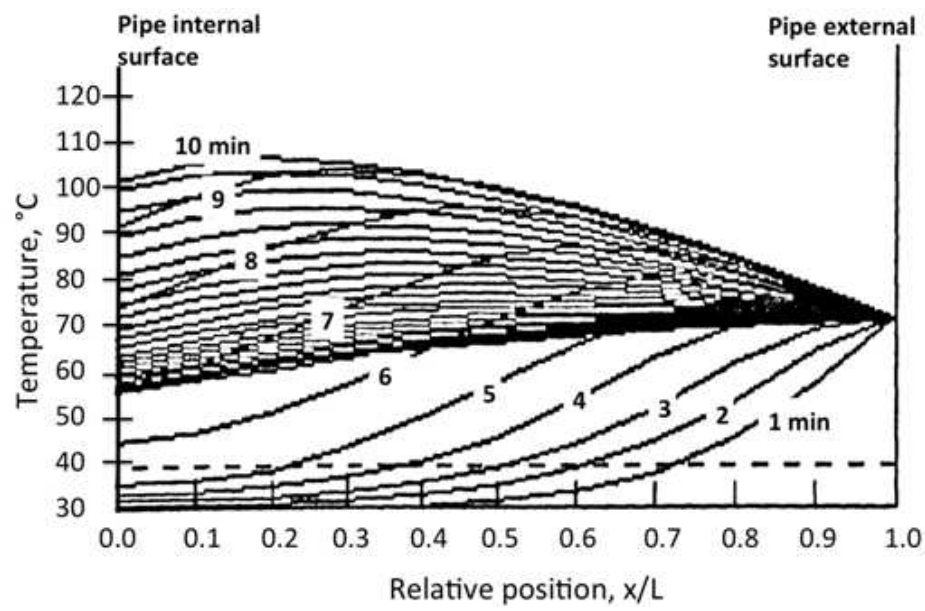

Fig. 2. Temperature profile through the pipe wall thickness calculated for a $2 \mathrm{~cm}$ wall thickness (only first 10 curved are numbered after that temperature profiles decreased to lower temperatures)

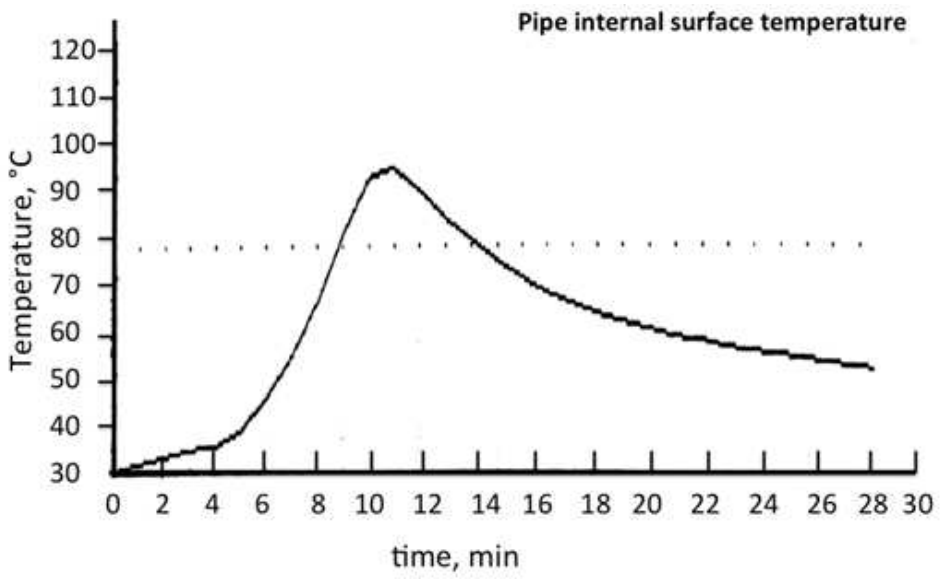

Fig. 3. Simulation results of the internal pipe wall surface temperature progress during centrifugal processing 


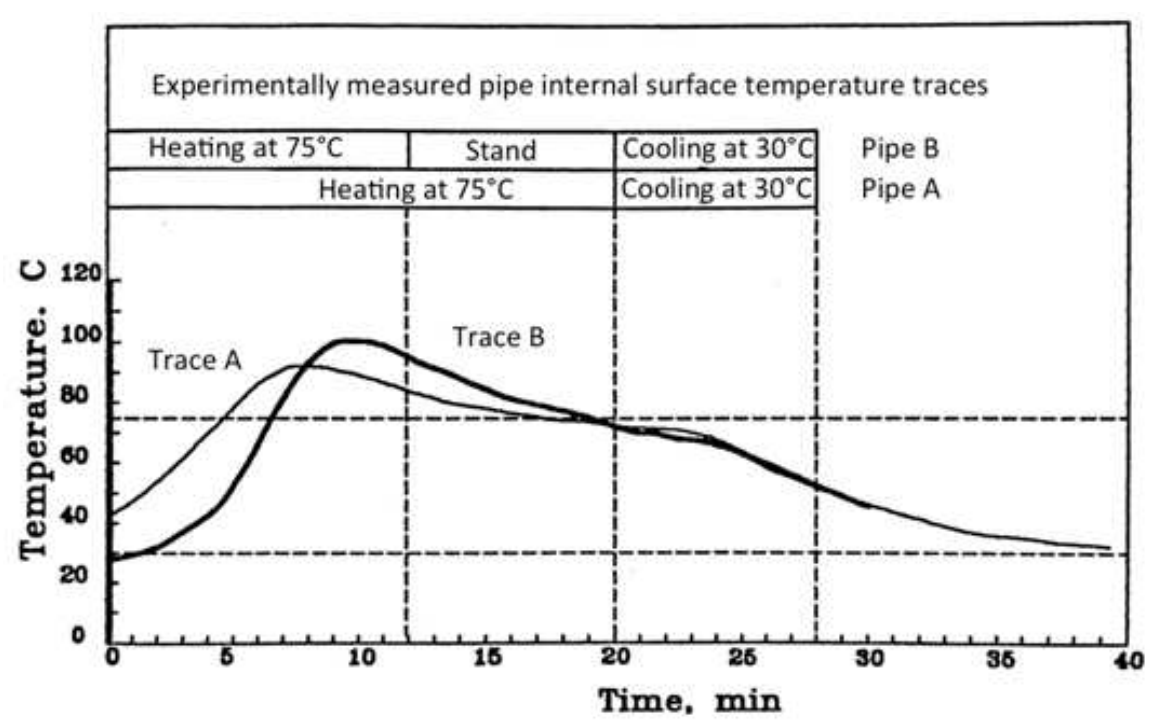

Fig. 4. Temperature changes measured at the internal surface of $2 \mathrm{~cm}$ wall thickness DN 600 pipes processed without $($ Trace $A)$ and with the self controlled heating regime (Trace B)

We have then experimentally tested the same processing condition of the simulation to the actual fabrication process of a centrifuged pipe $A$. In particular, the heating was from the outside with continuous hot water $\left(75^{\circ} \mathrm{C}\right)$ circulation for $20 \mathrm{~min}$ followed by $8 \mathrm{~min}$ of cooling $\left(30^{\circ} \mathrm{C}\right.$ water circulation).

The temperature at the internal surface was monitored with an optical pyrometer and it is reported in Figure 4 as Trace $A$ that well fitted with our previous simulation results reported in Fig. 3.

In particular our simulations predicted that a maximum temperature of $100^{\circ} \mathrm{C}$ was reached at the internal surface just after $10 \mathrm{~min}$ and slowly decreased as the external surface was being heated (by hot water at $75^{\circ} \mathrm{C}$ ).

During the first $10 \mathrm{~min}$ the water circulating at $75^{\circ} \mathrm{C}$ was subtracting heat from pipe $\mathrm{A}$, that would have caused the pipe to reach a higher internal temperature.

By applying to the pipe $B$ fabrication process the combined monitoring (pyrometer) and thermo kinetic and chemo rheological control simulation algorithm, it was observed that it stopped the heating after $12 \mathrm{~min}$ in order to permit the system self-heating.

We have experimentally verified, see pyrometer Trace $B$ on Fig. 4, that the temperatures reached in the self controlled heating cycle (comprising of a $12 \mathrm{~min}$ hot water circulation, a stop of $8 \mathrm{~min}$ and subsequent final 8 min cooling) were higher than for pipe $A$ even if the system was initially at a lower temperature (this was due to a change of the environmental temperature). The monitoring and control system, other than chose the more adequate heating cycle, has also correctly modified the input parameters to the better respond to the variation of the initial condition.

The implementation of the control code by also considering the heat flux at the pipe external surface in contact with the thermo stated water jacketed will further improve the system ability to find still more convenient process conditions.

A calorimetric characterization done on samples taken from the two differently processed pipes confirmed a high level of polymerization and glass transition temperatures of the pipe $B$, which was processed with an even shorter and less energy consuming heating cycle.

The level of polymerization measured on samples taken from pipes $\mathrm{A}$ and $\mathrm{B}$ processed at different conditions without and with the process monitoring and control was, indeed, significantly different. The thermo calorimetric analysis (DSC) indicated that the mean level of polymerization for the two pipes was different and differences through the pipe wall thickness were also measured.

In particular, the resin polymerizations levels for the standard (continuous $75^{\circ} \mathrm{C}$ water circulation for $20 \mathrm{~min}$ ) and self controlled processes $\left(12\right.$ min reduced $75^{\circ} \mathrm{C}$ water circulation) were 85 and $90 \%$ in the center of the pipes wall and 89 and $95 \%$ at the inner surface, respectively.

These calorimetric data confirmed the significant improvement resulting from the adoption of a monitoring and intelligent control algorithm.

\section{Conclusion}

Society, Science and Technology share cyclical relationships that, while mutually impacting and influencing each other, help factories to answer to progressively growing quality customer requirements.

This new approach will respond to the growing industrial expectation for an increased efficiency and productivity, but it will also address social needs by implementing interactive collaboration between humans and machines (Hwang et al., 2016). 
The present papers have shown in this case study how an interactive production system can improve product quality while implementing production strategies for specific technological problems.

In particular, the incomplete cure in different sections of underground waste water pipes wall may be responsible of a slow creep in some of these layers (especially for the central part of the pipe wall) once subjected to constant loading for long times. This was, in fact, one of the problems that can arise for not well processed GRP pipes. The creep characteristics, more than the glass transition temperatures, are sensible to the level of polymerization reached during the process and subsequent storage. An increase of the creep resistance was in fact measured after post-curing of the material.

The choice to use of a more reactive initiator (AAP versus MEKP) provided a better level of polymerization and, hence, an improved creep resistance of the material.

It has been developed a decisional algorithm to define the evaluation criteria to establish the relationship between the resin reactivity, pipe wall thickness and temperature and time of heating to improve the composite pipes reliability.

The thermo-rheological model and the calculation code discussed in this report, uses information's about the kinetic of polymerization and heat generation coupled with the thermal exchange to predict the temperature profiles and level of cure as a function of processing time and position. When interfaced to an active monitoring and control system such process optimization algorithm may improve the quality and reliability of these composites manufacture.

Concluding, the modeling of the system adequately describes the thermal behavior of system of known reactivity in different conditions of processing and pipe geometry. In particular, the heating heating/stop and cooling procedures could be optimized for pipes of different wall thickness or for systems containing different initiators or different amount of inhibitor in changing external environmental conditions.

\section{Acknowledgement}

This text was acknowledged and appreciated by Assoc. Pro. Taher M. Abu-Lebdeh, North Carolina A and T State Univesity, United States, Samuel P. Kozaitis, Professor and Department Head at Electrical and Computer Engineering, Florida Institute of Technology, United States.

\section{Author's Contributions}

All the authors contributed equally to prepare, develop and carry out this manuscript.

\section{Ethics}

This article is original and contains unpublished material. The corresponding author approved the manuscript and confirms that no ethical issues involved. Authors declare that are not ethical issues and no conflict of interest that may arise after the publication of this manuscript.

\section{References}

Apicella, A., L. Nicolais and J.C. Halpin, 1983. 28th National SAMPE meeting on "Materials and Process Continuing Innovation". Anheim, USA.

Apicella, A., L. Nicolais, M. Iannone and P. Passerini, 1984. Thermokinetics and chemorheology of the cure reactions of the tetraglycidyl diamino diphenyl methane-diamino diphenyl sulfone epoxy systems. J. Applied Polymer Sci., 29: 2083-2096.

DOI: 10.1002/app.1984.070290616

Apicella, A., 1986. Influence of Chemorheology on the Epoxy Processing. In: Developments in Reinforced Plastics-5: Processing and Fabrication, Pritchard, G.

Bamford, C.H., W.G. Barb, A.D. Jenkins and P.R. Onyon, 1958. The Kinetics of Vinyl Polymerization by Radical Mechanisms. Academic Press, New York, pp: 318.

Beisemberg, J.A. and D.H. Sebastian, 1983. Principles of Polymerization Engineering. 1st Edn., Wiley, New York, ISBN-10: 0471086169, pp: 744.

Castro, J.M. and C.W. Macosko, 1982. Studies of mold filling and curing in the reaction injection molding process. AIChE J., 28: 250-260. DOI: $10.1002 /$ aic.690280213

Cuadrado, T.R., J. Borrajo, R.J.J. Williams and F.M. Clara, 1983. On the curing kinetics of unsaturated polyesters with styrene. J. Applied Pol. Sci., 28: 485-499. DOI: 10.1002/app.1983.070280205

Fan, J.D. and L.J. Lee, 1986. Optimization of polyester sheet molding compound. Part II: Theoretical modeling. Polym. Compost., 7: 250-260.

Flory, P., 1953. Principles of polymer chemistry. 1st Edn., Cornell University Press, Ithaca, ISBN-10: 0801401348, pp: 672.

Halpin, J.C., J.L. Kardos and P.P. Dudukovic, 1983. Processing science: An approach for prepreg composite systems. Pure Applied Chem., 55: 893-906. DOI: $10.1351 /$ pac198355050893

Han, H. and K.W. Lem, 1983. Chemorheology of thermosetting resins. I. The chemorheology and curing kinetics of unsaturated polyester resin. J. Applied Pol. Sci., 28: 3155-3183.

DOI: 10.1002/app.1983.070281014

Han, C.D. and D.S. Lee, 1987. Analysis of the curing behavior of unsaturated polyester resins using the approach of free radical polymerization. J. Applied Polym. Sci., 33: 2859-2876.

DOI: $10.1002 /$ app.1987.070330820

Huang, Y.J. and L.J. Lee, 1976. AICHE J., 22: 268-268. 
Hwang, G., J. Lee, J. Park and T.W. Chang, 2016. Developing performance measurement system for Internet of Things and smart factory environment. Int. J. Product. Res.

DOI: $10.1080 / 00207543.2016 .1245883$

Kamal, M.R., S. Sourour and M. Ryan, 1973. Integrated thermo-rheological analysis of the cure of thermosets. SPE ANTEC Tech. Papers, 19: 187-191.

Kaštánek A, Bohdanecký M, 1985, A viscometric study of dilute solutions of low-molecular-weight unsaturated polyesters. Eur. Polymer J., 21: 1021-1025. DOI: 10.1016/0014-3057(85)90207-1

Kenny J.M., A. Apicella, L. Nicolais, 1989. A model for the thermal Chemorheological behavior of thermosets I: Processing of Epoxy based composites. Polym Eng. Sci., 29: 973-983.

DOI: $10.1002 /$ pen.760291502
Lee, L.J., 1981. Curing of compression molded sheet molding compound. Polym. Eng. Sci., 21: 483-492. DOI: $10.1002 /$ pen.760210808

Lee, D.S. and C.D. Han, 1987. A chemorheological model for the cure of unsaturated polyester resin. Polym. Eng. Sci., 27: 955-963. DOI: $10.1002 /$ pen. 760271306

Nicolais, L. and A. Apicella, 1985. Processing of Composite structures. Pure Applied Chem., 57: 1701-1706. DOI: 10.1351/pac198557111701

Odian, G., 1970. Principles of Polymerization. 1st Edn., McGraw-Hill, New York, pp: 652.

Pusatcioglu, S.Y., A.L. Fricke and J.C. Hassler, 1979. Heats of reaction and kinetics of a thermoset polyester. J. Applied Pol. Sci., 24: 937-946. DOI: 10.1002/app.1979.070240407 